\title{
Ultrafast biexciton dynamics in a ZnO thin film
}

\author{
Fang-Yi Jen, Yen-Cheng Lu, Cheng-Yen Chen, Hsiang-Chen Wang, and C. C. Yang ${ }^{\text {a) }}$ \\ Graduate Institute of Electro-Optical Engineering and Department of Electrical Engineering, National \\ Taiwan University, 1, Roosevelt Road, Sec. 4, Taipei, Taiwan, Republic of China

\begin{abstract}
Bao-ping Zhang and Yusaburo Segawa
Photodynamics Research Center, RIKEN (the Institute of Physical and Chemical Research), Sendai, Japan
\end{abstract}

(Received 11 May 2005; accepted 11 July 2005; published online 8 August 2005)

\begin{abstract}
The emission lines of biexciton and donor-bound biexciton are observed in a high-quality $\mathrm{ZnO}$ thin-film sample with time-resolved photoluminescence (TRPL) measurement. The TRPL intensity profiles reveal the formation sequence of various types of exciton. After free excitons are first generated, part of them is trapped by neutral donors to form donor-bound excitons. The other part contributes to the generation of biexcitons through free exciton scattering. Next, a donor-bound biexciton is generated through the trapping of a biexciton or two free excitons by a neutral donor or the trapping of a free exciton by a donor-bound exciton. Except donor-bound exciton, the relaxations of all other exciton states show two decay stages. Either the increasing or decreasing trends of the calibrated decay times in increasing the excitation power are well interpreted with a four-level model. (C) 2005 American Institute of Physics. [DOI: 10.1063/1.2012539]
\end{abstract}

Because of its large exciton binding energy at about 60 $\mathrm{meV}, \mathrm{ZnO}$ has attracted much attention in crystal growth and optical property characterization. With such a large exciton binding energy, the dominance of exciton recombination in the radiative process, even up to the room temperature, results in the high photon emission efficiency. Recently, besides the nanostructures, such as nanotubes, nanorods, and nanowalls, high-quality $\mathrm{ZnO}$ thin films have been grown by molecular-beam epitaxy, ${ }^{1,2}$ metalorganic chemical vapor deposition (MOCVD), ${ }^{3,4}$ and sputtering. ${ }^{5}$ Meanwhile, the growths of $\mathrm{ZnO} / \mathrm{MgZnO}$ quantum-well structures ${ }^{6,7}$ and $\mathrm{ZnSO}$ and $\mathrm{ZnCoO}$ ternary compounds ${ }^{8,9}$ have been implemented. For device fabrication, $p$-type $\mathrm{ZnO}$ (Refs. 10 and 11) and $\mathrm{ZnO}$ grown on $\mathrm{GaN}$ (Ref. 12) have been the issues of interest.

Normally, in photoluminescence (PL) measurement with low excitation, the emission of donor-bound exciton $\left(D^{0} X\right)$ dominates the spectrum at low temperatures $(<60 \mathrm{~K})$ and that of free exciton $(F X)$ dominates at high temperatures. If the acceptor density is high in a sample, the feature of donoracceptor pair (DAP) can be observed. In the case of high excitation, the biexciton $(X X)$ feature can be observed in a high-quality $\mathrm{ZnO}$ sample. ${ }^{13,14}$ Although the biexciton dynamics in other materials were studied, ${ }^{15-17}$ that in $\mathrm{ZnO}$ has not been well explored. In particular, the interplay between $X X, D^{0} X$, and $F X$ in $\mathrm{ZnO}$ has never been investigated.

In this letter, we study the ultrafast biexciton dynamics in a $\mathrm{ZnO}$ thin-film sample with time-resolved PL (TRPL) measurement. In the measurement, not only the emission line (the $M$ line) of $X X$ is observed, but also that (the $D^{0} M$ line) of donor-bound biexciton $\left(D^{0} X X\right)$ can be clearly seen. The calibrated two-stage decay times are used to build the model of ultrafast biexciton dynamics in such a $\mathrm{ZnO}$ sample. The $\mathrm{ZnO}$ thin film was grown with MOCVD on a (0001) sapphire substrate with 6 Torr in pressure at $450{ }^{\circ} \mathrm{C}$ for $90 \mathrm{~min}$. The temporal resolution in the TRPL measurment is 19 ps.

${ }^{\text {a) Electronic mail: ccy@cc.ee.ntu.edu.tw }}$
The excitation photon energy is $3.444 \mathrm{eV}(360 \mathrm{~nm})$, which is higher than the $F X$ energy level at $3.378 \mathrm{eV}$.

Figure 1 shows the time-integrated PL spectra at $10 \mathrm{~K}$ with several excitation powers. The focused excitation laser beam size on the sample was estimated to be $25 \mu \mathrm{m}$. Therefore, the average power of $9.05 \mathrm{~mW}$ corresponds to the average intensity of $1.93 \mathrm{~kW} / \mathrm{cm}^{2}$, which is in the same order of magnitude as those for observing biexciton in $\mathrm{ZnO}{ }^{13} \mathrm{At}$ low excitation, a major peak at $3.37 \mathrm{eV}\left(D^{0} X\right)$ and a minor peak at $3.306 \mathrm{eV}$ (DAP) can be observed. As the excitation power increases, two more major peaks at 3.3614 and 3.353 $\mathrm{eV}$ can be identified. Also, the DAP peak blueshifts with increasing excitation power. ${ }^{13}$ Figure 2 shows the integrated PL intensities of the three major peaks as functions of excitation power in the log-log scale. The calibrated slopes of the three sets of data points at around $0.93,1.27$, and 1.53 are shown in the legend. The set with the slope of 0.93 has been identified as the feature of $D^{0} X$. The other two sets of superlinear slopes are related to the activities of biexcitons and bound-biexcitons. ${ }^{18,19}$ With the spectral peak position at $3.3614 \mathrm{eV}$, the central major peak corresponds to the emis-

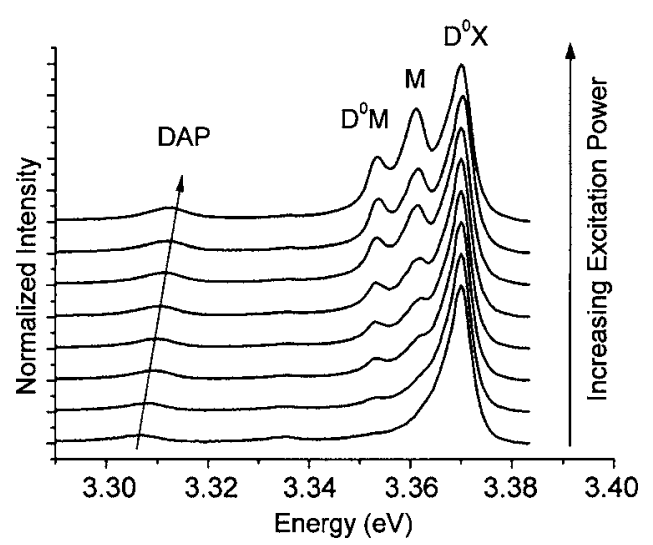

FIG. 1. Time-integrated PL spectra at $10 \mathrm{~K}$ with several average excitation powers at $1.147,3.56,7.76,9.05,11.45,15.33,19.11$, and $27.02 \mathrm{~mW}$. The excitation photon energy is $3.444 \mathrm{eV}$. 


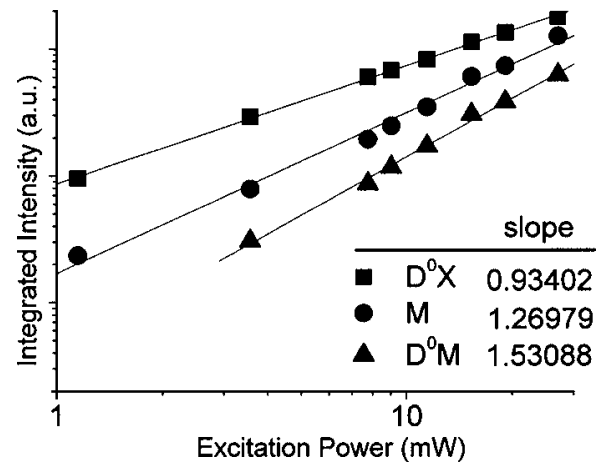

FIG. 2. Time-integrated PL intensities as functions of average excitation power at three photon energies corresponding to the emissions of $D^{0} X, X X(M)$, and $D^{0} X X\left(D^{0} M\right)$.

sion of $X X$, which is usually called the $M$ line. ${ }^{13}$ In such an emission process, one of the electron-hole pairs of an $X X$ recombines to release a photon and an $F X$. The left major peak at $3.353 \mathrm{eV}$ corresponds to the emission of $D^{0} X X$, which is designated as the $D^{0} M$ line. ${ }^{18}$ The relative energy levels of those exciton states are depicted in Fig. $3 .{ }^{20}$ Here, we follow the convention and define the binding energies, $G_{M}, G_{D^{0} M}$, and $G_{D^{0} X}$, corresponding to the biexciton binding energy, donor binding energy of biexciton, and donor binding energy of exciton, respectively. In our measurement, the three binding energies are $G_{M}=16.6 \mathrm{meV}, G_{D^{0} M}$ $=16.4 \mathrm{meV}$, and $G_{D^{0} X}=8 \mathrm{meV}$. Those values are quite consistent with what reported previously. ${ }^{18}$

Figure 4 shows the TRPL intensity profiles at the spectral peaks of $D^{0} X, M, D^{0} M$, and $F X$ at $10 \mathrm{~K}$. The excitation power is $27.02 \mathrm{~mW}$. Although the $F X$ feature is unclear in Fig. 1 (a very minor side lobe), its energy level has been well identified $(3.378 \mathrm{eV})$. In Fig. 4, the dashed fitting lines for the data curves in the log scale are plotted to demonstrate the two-stage decay characteristics of $M, D^{0} M$, and $F X$. It is interesting to note the large difference in decay slope between the two stages of $F X$. Also, the slower decay in the first stage, compared with that of the second stage, of $D^{0} M$ shows the unique behavior of $D^{0} X X$. The relative time delays of the maximum intensities of $F X, D^{0} X, M$, and $D^{0} M$ are 0 , $14.31,14.31$, and $30.18 \mathrm{ps}$, respectively. These results indicate the generation sequence of different types of exciton. $F X$ s are first generated after excitation. Then, part of them is trapped by neutral donors to form $D^{0} X$ s. Also, an $F X-F X$

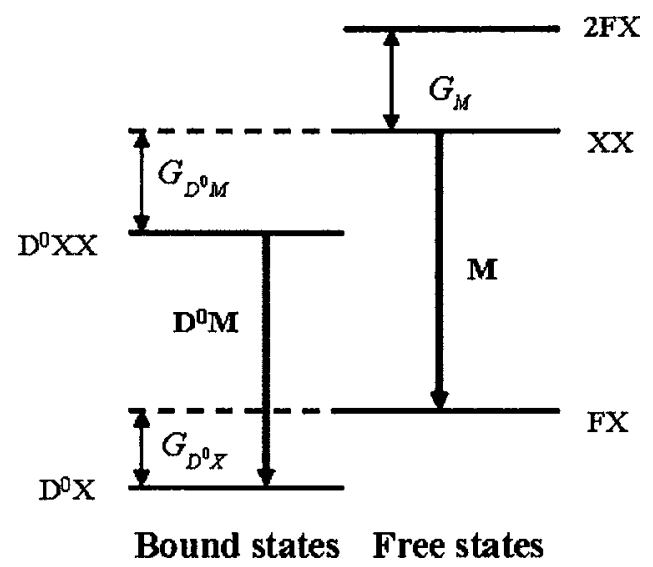

FIG. 3. Schematic demonstration of the relative energy levels of various exciton states.

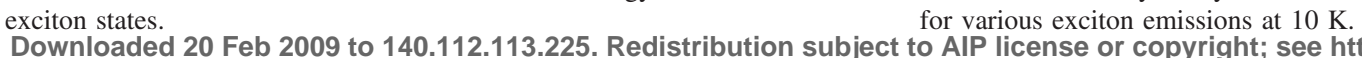

FIG. 5. PL intensity decay times as functions of average excitation power

FIG. 4. TRPL intensity profiles of four photon energies corresponding to the emissions of $F X, D^{0} X, X X(M)$, and $D^{0} X X\left(D^{0} M\right)$ at $10 \mathrm{~K}$.

scattering process results in the generation of $X X$ s. These two mechanisms have about the same time scale (14.31 ps). Next, the generation of $D^{0} X X$ may have three channels. First, the trapping of an $X X$ by a neutral donor can lead to the formation of a $D^{0} X X$. Second, the trapping of an $F X$ by a $D^{0} X$ can result in a $D^{0} X X$. Third, the trapping of two $F X$ s by a neutral donor can also produce a $D^{0} X X$ although the probability of such a process is quite low. With these processes, the formation of $D^{0} X X$ is further delayed by a time scale of about 16 ps.

Figure 5 shows the calibrated decay times of the four types of exciton as functions of excitation power. Among those curves, we first notice the short first-stage decay times of $F X$. This fast decay ( 40 ps time scale) is attributed to the formations of $D^{0} X$ and $X X$ through the trapping and scattering processes, respectively. The slow down of the $F X$ decay after the delay of about 70 ps (see Fig. 4), i.e., the slower second-stage decay (120 ps time scale), is due to the supply through the $M$ and $D^{0} M$ emissions. The recombination of one of the electron-hole pairs in an $X X$ can lead to the generation of an $F X$. Therefore, one can see the increasing trend of the second-stage decay time with increasing excitation power ( $>10 \mathrm{~mW}$ in Fig. 5). On the other hand, the decreasing trend of decay time in the first stage of $F X$ relaxation originates from the increasing $X X$ generation as the excitation power increases. However, the weak dependence of $F X$ decay time on excitation power, in either the first- or secondstage decay, implies that the formation of $D^{0} X$ plays a significant role in $F X$ relaxation.

The increasing trend of $D^{0} X$ emission decay time with excitation power can be interpreted as the significant supply from $F X$ (relaxed from $X X$ ) and $D^{0} X X$ through their relaxations. At $10 \mathrm{~K}, D^{0} X$ is the most stable state and all the

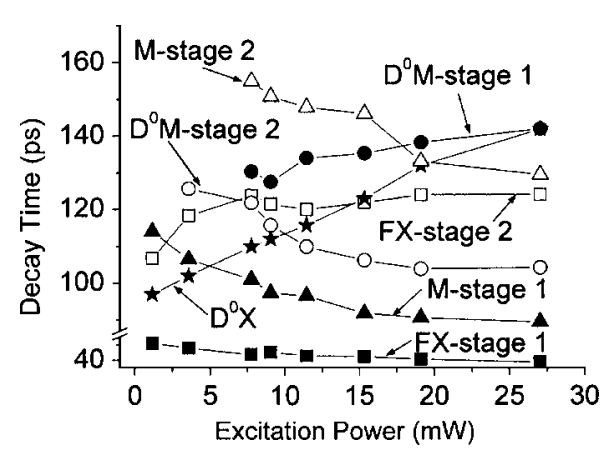

AIP license or copyright; see http://apl.aip.org/apl/copyright.jsp 
relaxations of $F X, X X$, and $D^{0} X X$ end up with the supply to $D^{0} X$. The decay times of both the faster first-stage and the slower second-stage decays of $X X$ (the $M$ line) decrease with excitation power. The faster first-stage decay is due to the relaxation of $X X$ into the $D^{0} X X$ state. The slowdown of the second-stage $X X$ relaxation can be attributed to the saturation of $D^{0} X X$ formation. It is noted that the major supply to the $X X$ population through the $F X-F X$ scattering occurs only in the very early stage $(<70 \mathrm{ps})$, which is earlier than the temporal range for the decay time calibration of the $M$ line. The decreasing trends of the $M$-line intensity with excitation power originate from the higher radiative recombination rate of $X X$ than that of $F X .^{16,17,21}$ In a case of high excitation power, a higher fraction of total carriers exists in the form of $X X$ such that in the $X X-F X$ cycling process, the effective decay rate of the total carrier density becomes higher, when compared with that of a lower excitation case. Such a higher decay rate is observed as the shorter decay times of the $M$-line intensity. The behavior of $D^{0} X X$ is quite different. First, the first-stage decay time of the $D^{0} M$ line is longer than that of the second stage. Second, the decay time of the first stage increases with excitation power although that of the second stage shows the opposite trend. The slower firststage decay can be attributed to the significant supply from $X X$ and $D^{0} X$. The increasing decay time with excitation power in this stage is simply due to the more supply as the excited carrier density (and hence the $X X$ density) is increased. After this supply process (the first stage of the M line) is slowed down, the decay time in the second stage is expected to decrease with excitation power, like the case of the $M$ line.

In summary, we have performed the TRPL measurement on a high-quality $\mathrm{ZnO}$ thin-film sample and observed the emission lines of $X X$ and $D^{0} X X$. The TRPL intensity profiles indicated the formation sequence of different types of exciton. After $F X$ s were first generated, part of them was trapped by neutral donors to form $D^{0} X \mathrm{~s}$. The other part became $X X \mathrm{~s}$ through $F X-F X$ scattering. Next, a $D^{0} X X$ could be generated through the trapping of an $X X$ or two $F X$ s by a neutral donor or the trapping of an $F X$ by a $D^{0} X$. Except $D^{0} X$, all other exciton states showed two decay stages in their relaxations. Either the increasing or decreasing trends of the calibrated decay times in increasing the excitation power were well interpreted with a four-level model.

This research was supported by National Science Council of Taiwan, The Republic of China, under Grant Nos. NSC 93-2210-M-002-006 and 94-2215-E-002-015, and by U.S. Air Force under Contract Nos. AOARD-04-4026 and AOARD-05-4085.

${ }^{1}$ Y. Chen, D. Bagnall, and T. Yao, Mater. Sci. Eng., B 77, 190 (2000).

${ }^{2}$ D. C. Look, D. C. Reynolds, C. W. Litton, R. L. Jones, D. B. Eason, and G. Cantwell, Appl. Phys. Lett. 81, 1830 (2002).

${ }^{3}$ K. Haga, T. Suzuki, Y. Kashiwaba, H. Watanabe, B. P. Zhang, and Y. Segawa, Thin Solid Films 433, 131 (2003).

${ }^{4}$ T. Gruber, C. Kirchner, K. Thonke, R. Sauer, and A. Waag, Phys. Status Solidi A 192, 166 (2002).

${ }^{5}$ S. F. Chichibu, T. Yoshida, T. Onuma, and H. Nakanishi, J. Appl. Phys. 91, 874 (2002).

${ }^{6}$ B. P. Zhang, N. T. Binh, K. Wakatsuki, C. Y. Liu, Y. Segawa, and N. Usami, Appl. Phys. Lett. 86, 032105 (2005).

${ }^{7}$ T. Makino, C. H. Chia, N. T. Tuan, H. D. Sun, Y. Segawa, M. Kawasaki, A. Ohtomo, K. Tamura, and H. Koinuma, Appl. Phys. Lett. 77, 975 (2000).

${ }^{8}$ B. K. Meyer, A. Polity, B. Farangis, Y. He, D. Hasselkamp, T. Krämer, C. Wang, U. Haboeck, and A. Hoffmann, Phys. Status Solidi C 1, 694 (2004).

${ }^{9}$ Y. Zheng, J. C. Boulliard, A. Soyer, and J. F. PeÂtroff, Acta Crystallogr., Sect. A: Found. Crystallogr. A60, 339 (2004).

${ }^{10}$ L. G. Wang and A. Zunger, Phys. Rev. Lett. 90, 256401 (2003).

${ }^{11}$ Y. R. Ryu, S. Zhu, D. C. Look, J. M. Wrobel, H. M. Jeong, and H. W. White, J. Cryst. Growth 216, 330 (2000).

${ }^{12}$ T. Gruber, C. Kirchner, R. Kling, F. Reuss, and A. Waag, Appl. Phys. Lett. 84, 5359 (2004).

${ }^{13}$ B. P. Zhang, N. T. Binh, K. Wakatsuki, Y. Segawa, Y. Kashiwaba, and K. Haga, Nanotechnology 15, S382 (2004).

${ }^{14}$ K. Hazu, T. Sota, K. Suzuki, S. Adachi, S. F. Chichibu, G. Cantwell, D. B. Eason, D. C. Reynolds, and C. W. Litton, Phys. Rev. B 68, 033205 (2003).

${ }^{15}$ M. Ikezawa and Y. Masumoto, Phys. Rev. B 53, 13694 (1996).

${ }^{16}$ G. Bacher, R. Weigand, J. Seufert, V. D. Kulakovskii, N. A. Gippius, A. Forchel, K. Leonardi, and D. Hommel, Phys. Rev. Lett. 83, 4417 (1999).

${ }^{17}$ K. Kyhm, R. A. Taylor, J. F. Ryan, T. Aoki, M. Kuwata-Gonokami, B. Beaumont, and P. Gibart, Phys. Status Solidi B 228, 475 (2001).

${ }^{18}$ A. Yamamoto, K. Miyajima, T. Goto, H. J. Ko, and T. Yao, Phys. Status Solidi B 229, 871 (2002).

${ }^{19}$ H. J. Ko, Y. F. Chen, T. Yao, K. Miyajima, A. Yamamoto, and T. Goto, Appl. Phys. Lett. 77, 537 (2000).

${ }^{20}$ M. Nakayama, H. Ichida, and H. Nishimura, J. Phys. C 11, 7653 (1999).

${ }^{21}$ J. C. Kim, D. R. Wake, and J. P. Wolfe, Phys. Rev. B 50, 15099 (1994). 